\title{
TOWARDS IMPROVED BIOAEROSOL MODEL VALIDATION AND VERIFICATION
}

\author{
BEN WILLIAMS $^{1}$, ENDA HAYES ${ }^{1}$, ZAHEER NASIR ${ }^{2}$, CATHERINE ROLPH $^{3}$, \\ SIMON JACKSON ${ }^{4}$, SHAGUN KHERA $^{4}$, ALAN BENNETT $^{5}$, TONI GLADDING ${ }^{3}$, \\ GILLIAN DREW ${ }^{2}$, JAMES LONGHURST ${ }^{1} \&$ SEAN TYRREL $^{2}$ \\ ${ }^{1}$ Air Quality Management Resource Centre, University of the West of England, UK \\ ${ }^{2}$ School of Water, Energy and Environment, Cranfield University, UK \\ ${ }^{3}$ Integrated Waste Systems, The Open University, Milton Keynes, UK \\ ${ }^{4}$ School of Biomedical and Healthcare Sciences, Plymouth University, UK \\ ${ }^{5}$ Public Health England, Salisbury, UK
}

\begin{abstract}
Bioaerosols, comprised of bacteria, fungi and viruses are ubiquitous in ambient air. Known to adversely affect human health, the impact of bioaerosols on a population often manifests as outbreaks of illnesses such as Legionnaires Disease and Q fever, although the concentrations and environmental conditions in which these impacts occur are not well understood. Bioaerosol concentrations vary from source to source, but specific industrialised human activities such as water treatment, intensive agriculture and open windrow composting facilitate the generation of bioaerosol concentrations many times higher than natural background levels. Bioaerosol sampling is currently undertaken according to the requirements of the Environment Agency's regulatory framework, in which the collection of bioaerosols and not its long-term measurement is of most importance. As a consequence, sampling devices are often moved around site according to changing wind direction and sampling intervals are invariably short-term. The dispersion modelling of bioaerosols from composting facilities typically relies on proxy pollutant parameters. In addition, the use of short term emission data gathering strategies in which monitors are moved frequently with wind direction, do not provide a robust reliable and repeatable dataset by which to validate any modelling or to verify its performance. New sampling methods such as the Spectral Intensity Bioaerosol Sensor (SIBS) provide an opportunity to address several gaps in bioaerosol model validation and verification. In the context of model validation, this paper sets out the current weaknesses in bioaerosol monitoring from the perspective of robust modelling requirements.
\end{abstract}

Keywords: bioaerosols, model validation, verification, dispersion modelling, monitoring.

\section{INTRODUCTION}

Since the introduction of the European Union Waste Framework Directive, 2008/96/EC, there has been a significant increase in the rate of recycled and composted waste. The Directive requires the United Kingdom (UK) and other Member States to recycle $50 \%$ of their Waste from Households (WfH) by 2020. Comprehensive data collected for the UK since 2010 show that recycling rates for the UK as a whole are at $40.4 \%$. Variability exists between devolved administrations, with Wales recycling 57.3\% of WfH and Scotland recycling $42.8 \%$ in 2016 [1]. The 2008/96/EC target for biodegradable municipal waste sent to landfill is $35 \%$ of a Nation's 1997 baseline (considered to be the total tonnage sent to landfill in 1997) [1]. In the UK's case this is equivalent to a reduction from the baseline of 21,460 Ktonnes, to 7,511 Ktonnes. In 2015 there were over 300 licensed composting facilities in the UK using a number of technologies including in-vessel and open composting systems. It is likely that this number will increase as more waste is diverted from landfill.

Bioaerosols, typically comprised of bacteria, fungi, and their components, are airborne microorganisms, released naturally into the air from the degradation of organic matter [2]. Ambient bioaerosol concentrations can be increased by anthropogenic activities, such as leaf blowing and the turning of composted garden waste. On an industrial scale, bioaerosols can 
be released from sewage plants during pre-treatment, aeration and sludge digestion [3], and from composting facilities during turning, screening and shredding activities [4]. Composting is the natural process of organic matter degradation by microorganisms. The more organic matter that is composted, the more micro-organisms will likely be present and consequently there is a potential for the release of a higher concentration of bioaerosols into the air.

Some bioaerosols are known to have adverse health effects, particularly for the immunecompromised. Brucellosis, Q Fever and Rift Valley fever are amongst the most well-known and are caused by exposure to Brucella spp, Coxiella burnettii and Phlebovirus respectively [5]. Due to the potential impact of bioaerosols on human health, the Environment Agency has determined that a site-specific risk assessment will be required for any composting facility that is within $250 \mathrm{~m}$ of any sensitive receptors (such as residential buildings), and they will need to demonstrate that bioaerosols are maintained at acceptable levels. These levels, as measured by the Association for Organics Recycling (AfOR) standard protocol, are 300, 1000 , and $500 \mathrm{cfu} \mathrm{m}^{-3}$ (colony forming units per metre cubed) for gram-negative bacteria, total mesophilic bacteria, and Aspergillus fumigatus respectively [6].

This short review of current bioaerosol monitoring and modelling approaches is conducted as part of a large-scale NERC funded research project to better understand the behaviour of bioaerosols emitted from open windrow composting facilities. The sampling devices used in the aforementioned research project and the means by which their data may be used in subsequent dispersion modelling activities are set out below.

Some progress has been made in characterising emissions from composting facilities, however relatively little headway has been made regarding the linked research questions of the modelling of bioaerosol dispersion; understanding exposure of the general public to bioaerosols; and setting health-based standards to quantify the associated health risk. A critical limiting factor in all of these areas is the lack of advanced microbiological methods (sampling, analytical, interpretative) to quantify and qualify bioaerosol emissions in real time and at high resolution. In this context, the following paper sets out the logic that underpins the dispersion modelling of bioaerosols and identifies areas where improvements may be made.

\section{AIR QUALITY MONITORING}

The following paragraphs review the conventional strategies used in air quality monitoring in order to highlight the differences between the monitoring of conventional pollutants and bioaerosols.

The UK established the world's first coordinated national pollution monitoring network, called the National Survey, in the wake of the 1956 Clean Air Act [7]. This survey primarily focused on the measurement of pollutants considered to be of industrial and domestic in origin, namely Black Smoke and Sulphur.

More recently, the monitoring network has been redesigned to assess direct and indirect emissions primarily from vehicle emissions including Particulate Matter, Nitrogen Dioxide and Ozone. The current system, known as the Automatic Urban Air Quality Network (AURN), operationally active since 1973, covers much of the UK with 146 current locations [8].

Standard measuring methods with minimum performance requirements for analysers have been produced through the European Committee for Standardisation (CEN) for monitoring air pollutants. These are set within the EU Ambient Air Quality Directive (2008/50/EC) and the amending Directive (EU) 2015/1480 [9].

At a national level, instrument type approval testing process is managed under the Monitoring Certification Scheme (MCERTS), under the stewardship of the UK Environment 
Table 1: UK AURN standard monitoring methods [10].

\begin{tabular}{|l|l|}
\hline Pollutant & Monitoring method \\
\hline Ozone $\left(\mathrm{O}_{3}\right)$ & UV Absorption \\
\hline $\begin{array}{l}\text { Nitrogen Oxides/ Nitrogen } \\
\text { Dioxide }\left(\mathrm{NO} / \mathrm{NO}_{2}\right)\end{array}$ & Chemiluminescence \\
\hline Sulphur Dioxide $\left(\mathrm{SO}_{2}\right)$ & UV Fluorescence \\
\hline Carbon Monoxide $(\mathrm{CO})$ & IR Absorption \\
\hline \multirow{5}{*}{ Particulate Matter $(\mathrm{PM})$} & $\begin{array}{l}\text { Tapered Element } \\
\text { Microbalance (TEOM) }\end{array}$ \\
\cline { 2 - 2 } & Beta Attenuation Monitor (BAM) \\
\cline { 2 - 2 } & Gravimetric Monitor \\
\cline { 2 - 2 } & FDMS \\
\hline
\end{tabular}

Agency. MCERTS performance standards mirror that of the CEN Standard Methods. Within the UK AURN the following techniques set out in Table 1, are used for different pollutants.

The reference method for the analysis of particulate matter is by gravimetry which does not allow for continuous, high resolution monitoring as it requires regular filter changes. As a consequence, continuous particulate matter monitors must demonstrate an equivalence with the reference method before use. An assessment of instrument equivalence performed by Bureau Veritas on behalf of Defra and the Devolved Administrations in which candidate instruments were tested to assess whether they met the equivalence criteria is publicly available [11].

Site specific monitoring methods for the determination of the concentrations of 'traditional' pollutants (considered herein as those set out within the 2008/50/EC Directive) in the UK are guided by the Environment Agency's Technical Guidance Note (monitoring) M8 [12]. Particular reference is made to the decision between intermittent or continuous sampling strategies, especially at sites in which site operations vary daily and consequently emissions may vary on a similar basis. Such sites include construction sites, landfills and composting facilities. Continuous particulate monitoring devices have been invaluable in allowing for the source apportionment of emissions as part of Detailed Assessments and model verification exercises [13].

There are no directives set for the monitoring of bioaerosols at a European level. Nationally, bioaerosol monitoring approaches are set out within the Environment Agency's M9 Technical Guidance Note on the Environmental Monitoring of Bioaerosols at Regulated Facilities, and in the AfOR protocol, and all instruments used in the collection of bioaerosols for reporting purposes are required to be MCERTS certified [6]. Within this protocol, the appropriate samplers to be used in the collection of bioaerosols are set out and the medium upon which samples are collected are presented. The protocol also sets out the appropriate monitoring locations, weather conditions and the timeframes over which samples should be collected. The purpose of these measurement guidelines is to identify the presence of viable

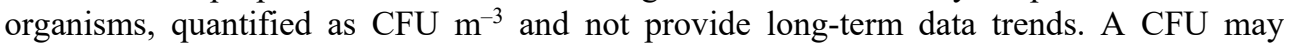
represent a single microbial cell or spore or a cluster that behaves as a single particle [6]. Commonly used monitoring instruments for the collection of bioaerosols are set out below.

The Institute of Occupational Medicine's (IOM) personal sampler has been used regularly for bioaerosol sampling [4], [14]. Compared with other bioaerosol samplers, they are inexpensive, lightweight and easily deployable in many locations. The IOM samplers 
typically run for 30 minutes at $2.2 \mathrm{~L} / \mathrm{min}$, with air drawn through a $0.8 \mathrm{~m}$ pore size polycarbonate filter [14]. The IOM sampling method allows for an understanding of bioaerosol concentrations over time and coupled with other sampling methods may provide valuable source term data.

Multi-stage Andersen impactors are frequently used to assess bioaerosol concentrations at various size fractions. The Andersen samplers are often fitted with a hemispherical baffle, and micro-organisms are collected onto either nutrient agar or tryptone soya agar (TSA) and malt extract agar (MEA) [15]. Andersen samplers are valuable sampling tools, particularly when using a multi-stage sampler as these will allow the collection and subsequent analysis of bioaerosols at different particle sizes. Coupled with IOM samplers, the multi-stage Andersen allows for the determination of specific bioaerosols and subsequently, through the analysis of each stage, their predominant particle size.

The purpose of the instruments set out above is to collect bioaerosols in a way that allows an understanding of concentrations, measured in CFU, over a designated, and typically short, timeframe. However, this approach does not allow for the assessment of long term trends in bioaerosol concentrations or facilitate an easy way of assessing variability of emissions based on site activity. Despite this, a relatively new real-time monitoring approach has the potential to overcome this challenge and is set out briefly below.

The Spectral Intensity Bioaerosol Sensor (SIBS) measures particle number, mass, volume and surface and the fluorescence intensity of particles across 16 wavelength intensities (from $288 \mathrm{~nm}$ to $735 \mathrm{~nm}$ ) to detect bioaerosols. The instrument allows for the determination of particle size, shape, asphericity and the measurement of fluorescence, which may indicate the presence of bioaerosols or other organic components at a high temporal resolution [16]. The continuous monitoring of particulate matter has provided significant benefits in source apportionment and model validation and verification at both a national and site-specific level. High resolution data from the SIBS may provide an understanding of site activities and bioaerosol concentrations thus contribute directly to the time-varying emission factors used in the modelling process. At present, data generated by the instrument does not allow for the clear identification of specific bioaerosols due to the complexity of interpreting the fluorescence data. Differentiation between bioaerosols and other organic fragments is also complex. However, in time, the SIBS could be used as widely as traditional continuous particulate matter samplers.

\section{AIR QUALITY MODELLING}

Spatially, air pollution can be modelled on a local, national, and global scale, depending on the pollutant and the modelling software being utilised. In the UK, air quality modelling is undertaken at a national and local level primarily to determine whether concentrations are in exceedance of limit values set down within the Ambient Air Quality Directive, (Directive 2008/50/EC (AAQD)), to assess emissions in line with the requirements of the National Emissions Ceiling Directive, (Directive 2016/2284 (ECD)) and also to assess emissions from specific industries and activities and their impact on local receptors. Nationally, the UK Government utilises the Pollution Climate Mapping model (PCM), a collection of models used to fulfil their requirements under the AAQD [17]. Locally, models such as ADMS, AERMOD and CALPUFF are used to assess concentrations from sources such as landfills, industry, agriculture and industrial composting facilities (there are other models for the assessment of road sources such as ADMS-Roads and ADMS-URBAN).

Specific guidance on modelling traditional air pollutants at a national scale and at a sitespecific level are presented in significant detail within the National Local Air Quality Management Technical Guidance (LAQM.TG(16)) [13]. For example, if modelling road 
traffic sources, clear guidelines on the data required for each model and the quality of the data expected are presented. These guidelines include specific information on the pollutant to be modelled, the source term data, time varying emissions and geographic parameters. As with all models, the quality of the data entered into them, drives the quality of the outputs.

In contrast to the above modelling approaches, bioaerosol modelling is still in its infancy. No commercially available model allows for the modelling of specific bioaerosols and as a consequence existing models have to be adapted. Douglas et al. [18] and CERC [19] note that ADMS models are most appropriate for assessing bioaerosol dispersion from composting facilities. Both sensitivity [18] and validation [20] studies have been undertaken to help inform model inputs, however these studies rely on limited monitoring datasets derived from short-term monitoring programmes designed to collect samples and not assess long term trends. As noted by Williams et al. [21] challenges in gathering relevant site-specific data for the modelling of bioaerosols exist. For example, IOM and Andersen samplers hold MCERTS accreditation and are consequently robust instruments to measure bioaerosols, however, their short term, low resolution sampling intervals do not allow for a continuous assessment of bioaerosol from a site. Furthermore, in order to underpin robust source-specific data gathering exercises, knowledge of the operating times of specific site activities is required. At some sites with clear and distinct operating periods for different activities this is relatively straightforward, however, on sites with less structured operating practices it is unlikely to capture the variability very well. The collection of meteorological data relevant to a particular site is also key to robust modelling and verification processes. Sites should have access to meteorological data from on-site weather stations, however where these are not accessible or comprehensive enough, meteorological data archived by the Centre for Environmental Data Analysis (CEDA) are available for research purposes [22]. Despite an inability of existing monitoring methods to collect robust long term, high resolution data suitable for modelling bioaerosols, new technologies such as the SIBS offer the potential for assessing long-term trends and providing robust data sets for model validation and verification.

\section{TOWARDS IMPROVED MODEL VERIFICATION AND VALIDATION}

Model verification and validation are often used interchangeably when referring to the assessment of modelling environmental systems. From a UK policy context, model validation is a controlled experimental design in which parameters are controlled, tested and the model design refined. Model verification is undertaken in real-world scenarios where many input parameters are unknown, but an understanding of the model performance is desired. The model design would not be altered by model verification, but input parameters can be altered/adjusted as appropriate [23], [24].

Oreskes et al. [25] argue that the validation and verification of models in natural systems is not possible due to poorly known and variable input parameters and inadequately understood or as yet unidentified detail. Whilst true of modelling most, if not all environmental systems, some fields are likely to have significantly more data than others to reduce the impact of these details on modelled outputs. Model verification can, in theory be undertaken with very basic monitoring data (i.e. one diffusion tube), model validation cannot be done without substantial, robust datasets. The confidence of both validation and verification is, however, enhanced by the quality of the monitoring data. For the validation and verification of traditional pollutants robust monitoring datasets are required. For example, for reporting to the $\mathrm{EC}$, instruments are required to meet internationally comparable standards. On a local level, MCERTS certified instruments are used for modelling purposes and where passive samplers are required (as in the case of $\mathrm{NO}_{2}$ ) robust monitor deployment 
and analysis protocols including bias adjustment are followed. Furthermore, when considering traffic pollutants or those derived from industrial facilities, the pollutant parameters and emission factors are known and can be well regulated.

As noted above, bioaerosol modelling is in its infancy and dispersion models do not include bioaerosols as a pre-loaded pollutant within their pollutant palates. As a consequence, bioaerosol modelling is undertaken using a proxy. In most bioaerosol modelling literature, bioaerosols are modelled using particulate matter [26], however others have argued that due to their relative size they could also be modelled as a gas [27]. Bioaerosol efflux parameters are similarly not well understood compared to those of traditional pollutants. Douglas et al. [18] noted that there are no reliable exit velocities of bioaerosol emissions from composting windrows, and an exit velocity of $2.95 \mathrm{~ms}^{-1}$ is considered appropriate. Temperature will also affect plume rise in the model and [18] modelling with a predetermined temperature of $29^{\circ} \mathrm{C}$ should be considered. Both exit velocity and temperatures set out here were considered by Douglas et al. [18] to be an average of the efflux parameters, falling between higher velocities and temperatures during the turning and mixing, and the relatively lower temperatures and exit velocities from non-agitated compost.

Bioaerosol monitoring strategies set out within the Environment Agency's bioaerosol monitoring guideline, M9, [28] do provide a robust sampling strategy, in particular, ensuring that monitoring is undertaken at the nearest sensitive receptor. As shown in Fig. 1, both upwind and downwind sites should be included in the sampling strategy, with downwind sites arranged in a fan shape.

Despite a robust sampling strategy, current monitoring regimes set out within M9 are designed to satisfy regulatory requirements for the collection of bioaerosols and are not conducive to modelling exercises. They suffice for basic low confidence verification studies but are not suitable for validation studies. For example, bioaerosol monitoring periods are very rarely longer than $1 \mathrm{hr}$ and more typically using IOM samplers 30 minutes long. Sampling using impaction methods such as the IOM and Andersen are constrained by the nature of the material caught and if samples are deployed for longer bioaerosols may begin to die. Douglas et al. [20] undertook a validation exercise of Aspergillus fumigatus in which incremental adjustments of model input parameters were made and the subsequently refined

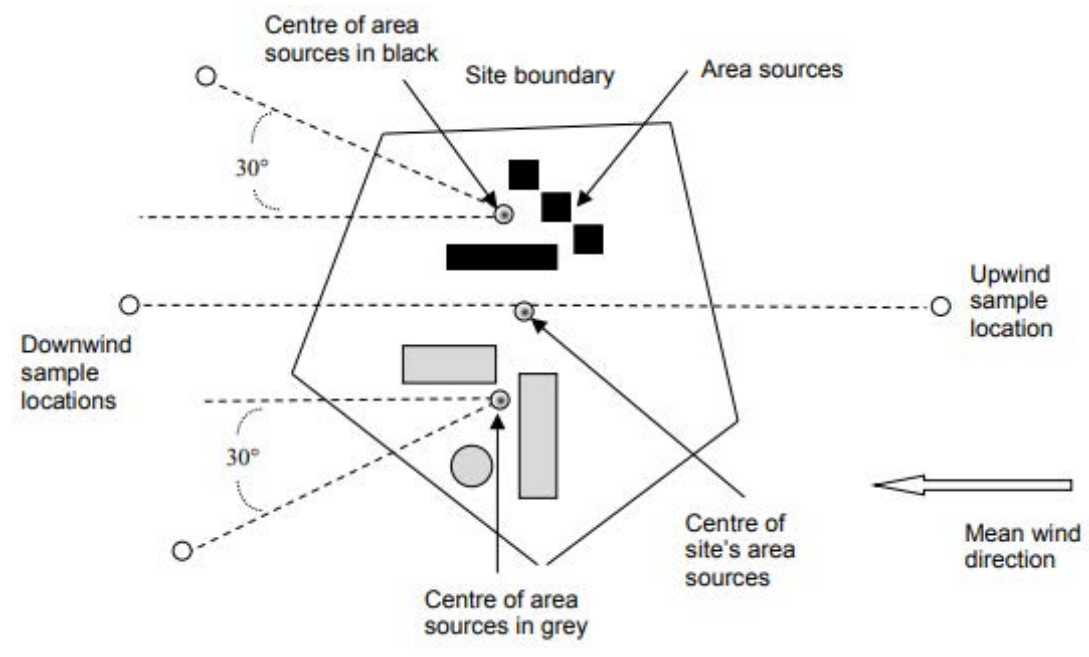

Figure 1: M9 bioaerosol sampling strategy. (Source: Environment Agency, 2017 [28].) 
model was validated against a 3-week dataset. It was noted that, amongst other external parameters, results were sensitive to the selection of either wet or dry deposition within the model parameters. Considering that current guidance advises collection of bioaerosols in dry conditions, this will inevitably be a significant limitation in any assessment using current methods.

Whilst these monitoring and modelling challenges constrain robust model validation and verification, there is an opportunity to maximise current instruments and new developments to improve this. For example, significant improvements could be achieved through research to determine a unit of mass per colony forming unit which will likely vary by specific bioaerosol.

Extended trials assessing emission rates from on-site composting activities such as shredding, screening and turning should be undertaken seasonally to complement that of Taha et al. [29]. Deployment of IOM samplers by Pankhurst et al. [30] allowed for the collection of bioaerosols downwind, however on subsequent sampling intervals when winds were blowing in other directions sampling locations were changed to capture the downwind bioaerosol concentration. For the purposes of model validation IOM samplers should be located down-wind of the predominant wind direction in a fixed location for the duration of the study, regardless of changes in wind direction and, if possible, the monitoring campaign should be extensive enough to account for seasonal changes.

Real time monitoring of bioaerosols for a prolonged monitoring period may allow for an understanding of long term trends in concentration. The SIBS will allow a high resolution, continuous bioaerosol sampling regime to be developed, however, at present, the instrument does not allow for the clear differentiation of bioaerosols from other organic matter in a simple, clear way. The SIBS would benefit from a bespoke study aimed at developing a bioaerosol emission inventory so that specific bioaerosols are clearly identifiable in environmental samples.

The determination of time-varying emission factors from each composting facility would aid in the refinement of the model and subsequent model validation and verification. Data can be gathered either through real-time monitoring or through the reliance on a site diary and inclusion of emission rates from previous studies [20], [29].

\section{DISCUSSION}

The European Waste Directive's drive to divert waste from landfill to recycling and composting facilities has resulted in the increase of such facilities across the UK over the last decade. Consequently, there will be an increase in the number of people living within close proximity to such facilities. This contribution has highlighted a number of key areas of bioaerosol modelling and monitoring that, if improved, could provide a significant advance in the robustness of bioaerosol modelling, allow for robust initial validation and subsequent verification.

Bioaerosol monitoring is currently undertaken with the aim of collecting bioaerosols for characterisation and consequently the datasets allow for model verification albeit with limited confidence in the results. Current bioaerosol monitoring methods are not suitable for validation exercises. Similarly, the monitoring methods set out in M9 preclude the use of devices in the rain and as a consequence the measurement of bioaerosols during such conditions is not undertaken. In doing so, wet deposition cannot be modelled, inhibiting the collection and temporal analyses of bioaerosols frequently.

The use of SIBS may provide a step change in bioaerosol monitoring, modelling and validation by allowing real-time high resolution continuous analysis of bioaerosol concentrations. It is hindered at present by a lack of clarity surrounding the monitored 
outputs, however, should research be undertaken to develop emission inventories there could be widespread uptake of the instrument for regulatory purposes.

As bioaerosol modelling is in its infancy it is unsurprising that models are yet to account for them within their pollutant palate. Should research provide advances in mass conversions of bioaerosols from CFU, it is likely that model developers will incorporate them into their packages.

\section{CONCLUSION}

In the context of model validation and verification, this contribution has identified the current monitoring and modelling methods used in the collection of bioaerosols and compared them with that expected of 'traditional' pollutants. Model validation and verification is explained and monitoring instrument quality requirements for traditional pollutants and bioaerosols set out. In the context of model validation and verification, current weaknesses with bioaerosol modelling and monitoring are described. Finally, several avenues for further research are described that would allow for improvements in modelling validation and verification.

\section{ACKNOWLEDGEMENT}

This work was funded by the Natural Environment Research Council (NERC) (reference number: NE/M011747/1).

\section{REFERENCES}

[1] Department for Environment Food and Rural Affairs, UK Statistics on Waste, 2018. https://assets.publishing.service.gov.uk/government/uploads/system/uploads/ attachment_data/file/683051/UK_Statisticson_Waste_statistical_notice_Feb_2018_ FINAL.pdf. Accessed on: 15 May 2018.

[2] Cox, C.S. \& Wathes, C.M., Bioaerosols in the environment. Bioaerosols Handbook, eds C.S. Cox \& C.M. Wathes, CRC Press: Boca Raton, FL, 1995.

[3] Li, J., Zhou, L., Zhang, X., Xu, C. \& Dong, L., Bioaerosol emissions and detection of airborne antibiotic resistance genes from a wastewater treatment plant. Atmospheric Environment, 124, pp. 404-412, 2016.

[4] Taha, M.P.M., Drew, G.H., Longhurst, P.J., Smith, R. \& Pollard, S.J.T., Bioaerosol releases from compost facilities: Evaluating passive and active source terms at a green waste facility for improved risk assessments. Atmospheric Environment, 40(6), pp. 1159-1169, 2006.

[5] Kanoute, Y.B., Gragnon, G.G., Schindler, C., Bonfoh, B. \& Schelling, E., Epidemiology of Brucellosis, Q Fever and Rift Valley Fever at the human and livestock interface in northern Cote d'Ivoire. Acta Tropica, 165, pp. 66-75, 2017.

[6] AfOR, The State of Composting and Biological Waste Treatment in the UK 2006/07, Association for Organics Recycling: Northamptonshire, Wellingborough, 2008.

[7] Department of Environment, Food and Rural Affairs, Monitoring Networks: Brief History. https://uk-air.defra.gov.uk/networks/brief-history. Accessed on: 17 May 2018.

[8] Department of Environment, Food and Rural Affairs, Automatic Urban and Rural Network. https://uk-air.defra.gov.uk/networks/network-info?view=aurn. Accessed on: 17 May 2018.

[9] Commission Directive (EU) 2015/1480 28 August 2015 on amending several annexes to Directives 2004/107/EC and 2008/50/EC of the European Parliament and of the Council laying down the rules concerning reference methods, data validation and location of sampling points for the assessment of ambient air quality, 2015. 
[10] Department of Environment, Food and Rural Affairs, EU Standard Methods for Monitoring and UK Approach. https://uk-air.defra.gov.uk/networks/monitoringmethods?view=eu-standards. Accessed on: 18 May 2018.

[11] Bureau Veritas, Assessment of UK AURN Particulate Matter Monitoring Equipment against the January 2010 Guide to Demonstration of Equivalence. Report No. AGG04003328/BV/AQ/DH/2658/V3, 2010.

[12] Environment Agency, Technical Guidance Note (Monitoring) M8: Monitoring Ambient Air, 2011.

[13] Department of Environment, Food and Rural Affairs, Local Air Quality Management Technical Guidance (TG16), 2018.

[14] Pankhurst, L.J. et al., Temporal and spatial changes in the microbial bioaerosol communities in green-waste composting. Federation of European Microbiology Societies Microbiology Ecology, 79, pp. 229-239, 2012.

[15] Taha, M.P.M., Drew, G.H., Tamer Vestlund, A., Aldred, D., Longhurst, P.J. \& Pollard, S.J.T., Enumerating actinomycetes in compost bioaerosols at source - use of soil compost agar to address plate 'masking'. Atmospheric Environment, 40(22), pp. 47594765, 2007.

[16] Nasir, Z.A. \& Tyrrel, S., Real-Time Bioaerosol Detection. AWE International, 2017. www.aweimagazine.com/article/real-time-bioaerosol-detection. Accessed on: 17 May 2018.

[17] Department of Environment, Food and Rural Affairs. Air Modelling for Defra. https://uk-air.defra.gov.uk/research/air-quality-modelling?view=modelling. Accessed on: 17 May 2018.

[18] Douglas, P. et al., Sensitivity of predicted bioaerosol exposure from open windrow composting facilities to ADMS dispersion modelling parameters. Journal of Environmental Management, 184(2), pp. 448-455, 2016.

[19] Cambridge Environmental Research Consultants. A review of the limitations and uncertainties of modelling pollutant dispersion from non-point sources. Contract report for the Atmospheric Dispersion Modelling Liaison Committee, 2015.

[20] Douglas, P. et al., Predicting Aspergillus fumigatus exposure from composting facilities using a dispersion model: A conditional calibration and validation. International Journal of Hygiene and Environmental Health, 220, pp. 17-28, 2017.

[21] Williams, B. et al., The challenges, uncertainties and opportunities of bioaerosol dispersion modelling from open composting facilities. Air Pollution XXV, WIT Press: Southampton and Boston, pp. 51-59, 2017.

[22] Centre for Environmental Data Analysis, MIDAS UK Hourly Weather Observation Data. http://catalogue.ceda.ac.uk/uuid/916ac4bbc46f7685ae9a5e10451 bae7c. Accessed on: 18 May 2018.

[23] Department of Environment, Food and Rural Affairs Evaluating the Performance of Air quality Models, 2010.

[24] Cambridge Environmental Research Consultants. Model Validation. www.cerc.co.uk/ environmental-software/model-validation.html Accessed on: 14 May 2018.

[25] Oreskes, N., Shrader-Frechette, K. \& Belitz, K., Verification, validation, and confirmation of numerical models in the earth sciences. Science, 263, pp. 641-646, 1994.

[26] Douglas, P. et al., Use of dispersion modelling for Environmental Impact Assessment of biological air pollution from composting: Progress, problems and prospects. Waste Management, 70, pp. 22-29, 2017. 
[27] Tamer Vestlund, A., Al-Ashaab, R., Tyrrel, S.F., Longhurst, P.J., Pollard, S.J.T. \& Drew, G.H., Morphological classification of bioaerosols from composting using scanning electron microscopy. Waste Management, 34, pp, 1101-1108, 2014.

[28] Environment Agency, Technical Guidance Note (monitoring) M8: Environmental monitoring of bioaerosols at a regulated facilities, 2017.

[29] Taha, M.P.M, Pollard, S.J.T., Sarkar, U. \& Longhurst, P., Estimating fugitive bioaerosol releases from static compost windrows: Feasibility of a portable wind tunnel approach. Waste Management, 25(4), pp. 445-450, 2005.

[30] Pankhurst, L.J. et al., Spatial variations in airborne microorganism and endotoxin concentrations at green waste composting facilities. International Journal of Hygiene and Environmental Health, 214, pp. 376-383, 2011. 